\title{
CIENAGA DE LA CAIMANERA: MANGLARES Y AVES ASOCIADAS
}

\section{SWAMP CAIMANERA: MANGROVE SWAMP AND BIRD ASSOCIATES}

URUETA, S. JUAN..$^{1 *}$ Biol., GARAY, S. CINDY. ${ }^{1}$ Biol., ZAMORA, G. ALEJANDRO. ${ }^{2}$ Esp., GALVAN-GUEVARA, SILVIA. ${ }^{3}$ Esp., DE LA OSSA, V. JAIME. ${ }^{4}$ Dr.

${ }^{1}$ Facultad de Educación y Ciencias, Universidad de Sucre, Colombia. ${ }^{2}$ Profesional especializado CARSUCRE. ${ }^{3}$ Grupo de Investigación en Biodiversidad Tropical, Maestría Ciencias Ambientales, Universidad de Cartagena. ${ }^{4}$ Profesor Titular Facultad de Ciencias Agropecuarias, Grupo de investigación en Biodiversidad Tropical, Universidad de Sucre, Colombia.

\section{*Correspondencia: jugus_0125@hotmail.com}

Recibido: 03-09-2010; Aceptado: 16-11-2010.

En la costa Caribe colombiana, existen ecosistemas marinos y costeros, que ofrecen una variedad de hábitat como playas, litorales, acantilados rocosos y manglares, que favorecen el establecimiento y la llegada de varias especies de aves acuáticas residentes y migratorias. Además, en esta región se encuentran ecosistemas de humedales aledaños a riberas de río, sabanas inundables, ciénagas de agua dulce y salobre, que se constituyen en hábitat importantes para la avifauna nacional (NARANJO, 1979; SÁNCHEZ-PÁEZ et al., 1997; INGEOMINAS, 1998).

La Ciénaga de la Caimanera es según (DÍAZ, 1997; BARRETO et al., 1999; ULLOA-DELGADO et al., 2001; FRANCO et al., 2005) una de las zona con mayor disponibilidad de hábitat como playas, manglar, planos lodosos y humedales, que existen en el sector, lo que la convierte en un sitio potencialmente importante para el desarrollo de un variado número de especies animales pertenecientes a todos los niveles taxonómicos de la cadena trófica. No obstante, por constituir los manglares parte estructural del área de estudio, en el presente documento solo se hará énfasis en la avifauna asociada a este ecosistema. En general, en estas formaciones boscosas, existen diversas formas de vida, desde bacterias autótrofas descomponedoras de materia orgánica, hasta mamíferos acuáticos pasando por un amplio número de grupos taxonómicos de invertebrados como anélidos, moluscos, artrópodos y vertebrados como peces, anfibios, reptiles, aves y algunos mamíferos terrestres que pasan por lo menos algún ciclo de su vida en la zona de manglar (SÁNCHEZ-PÁEZ et al., 2000).

Basado en los conceptos expuestos y atendiendo las necesidades de conservación e importancia de los manglares como hábitat fundamental de las aves, se realizó este trabajo con el fin de conocer la relación que existe entre la 
estructura y composición de la flora con la presencia de aves; debido a que son el componente zoológico más diverso y abundante asociado a este tipo de formación natural.

El estudio se realizó en la región Sur del Golfo de Morrosquillo, en el área conocida como Ciénaga de la Caimanera ubicada a 9²5'57.42" N y 75³7'53.64" $\mathrm{O}$, entre los meses de febrero y agosto del 2009; se implementaron visitas técnicas de inspección ocular en la ciénaga, con el fin de evaluar la estructura de los manglares y se registraron las aves asociadas a este tipo de formaciones boscosas utilizando el criterio presencia-ausencia descrito por DUNN et al., (2006).

Para el reconocimiento general de las especies de mangle existentes, se hizo una revisión secundaria de estudios previos (GIL-TORRES, 1998; SÁNCHEZPÁEZ et al., 2000; ULLOA-DELGADO et al., 2001; DÍAZ et al., 2008). Posteriormente se procedió al reconocimiento in situ de las especies de mangle mediante recorridos externos e internos en el bosque de manglar. De acuerdo con AGRÁZ-HERNÁNDEZ et al., (2006) se hizo colecta de muestras de las estructuras morfológicas de los mangles dependiendo del estado fenológico de las plantas, con el fin de diferenciar cada una de las especie existentes.

Según el MINISTERIO DEL MEDIO AMBIENTE (2002), para el departamento de Sucre se registran cuatro familias de mangles, representadas por cinco especies en todo el Caribe colombiano. Para la región sur del Golfo, se encontró solamente cuatro de las cinco especies reportadas para la costa Caribe colombiana. En general las especies de mangle observadas durante los distintos recorridos a través de la Ciénaga la Caimanera fueron: mangle rojo (Rhizophora mangle), mangle blanco (Laguncularia racemosa), mangle negro (Avicennia germinans) y mangle zaragoza (Conocarpus erecta), lo cual coincide con los resultados obtenidos en otros estudios sobre el estado de los manglares en Colombia (GIL-TORRES, 1998; SÁNCHEZ-PÁEZ et al., 2000; ULLOA-DELGADO et al., 2001; DÍAZ et al., 2008).

Durante el levantamiento de la estructura de la vegetación, se encontró, que la formación boscosa Ciénaga la Caimanera, presenta un manglar tipo borde de aproximadamente $500 \mathrm{~m}$ partiendo desde el caño principal en el sector Boca de la Ciénaga, lo que coincide con lo reportado por CARSUCRE (2004). En general, el mangle en esta primera zona es casi en su totalidad monoespecífico, con predominancia de mangle rojo (Rhizophora mangle), posteriormente se vuelve heterogéneo con codominio de esta especie, acompañado de mangle negro (Avicennia germinans) y en menor proporción mangle blanco (Laguncularia racemosa). Posterior a esta primera franja de manglar el bosque toma una estructura tipo cuenca, con zonas inundables en temporadas de lluvias, especialmente por los aportes hídricos de origen 
continental provenientes de los arroyos Petalaca y San Antonio. En esta zona, predomina mangle negro (Avicennia germinans) con presencia de mangle blanco (Laguncularia racemosa) y en menor proporción mangle rojo (Rhizophora mangle).

De igual forma se encontró que la presencia de estos arroyos permite el desarrollo de pequeñas franjas de manglares cuenca-ribereños, ubicados alrededor de los caños que transportan las aguas continentales al interior del manglar, caracterizados por la presencia de arboles de altura media de mangle rojo (Rhizophora mangle). El sector con mayor área de manglar es al Suroccidente de la Ciénaga, donde abunda mangle rojo (Rhizophora mangle), seguido por una asociación de este con mangle blanco (Laguncularia racemosa). En contraste, hacia el Oriente de la Caimanera, el arbolado de mangle se extiende $900 \mathrm{~m}$ aproximadamente desde el cuerpo de agua, con predominio de mangle negro (Avicennia germinans) en sentido Este - Oeste, seguido por una asociación de mangle negro (Avicennia germinans) con mangle blanco (Laguncularia racemosa) y algunos individuos de mangle rojo (Rhizophora mangle), terminando finalmente en una mancha de mangle rojo (Rhizophora mangle) circunscrita alrededor del espejo principal de de agua (Fig. 1).

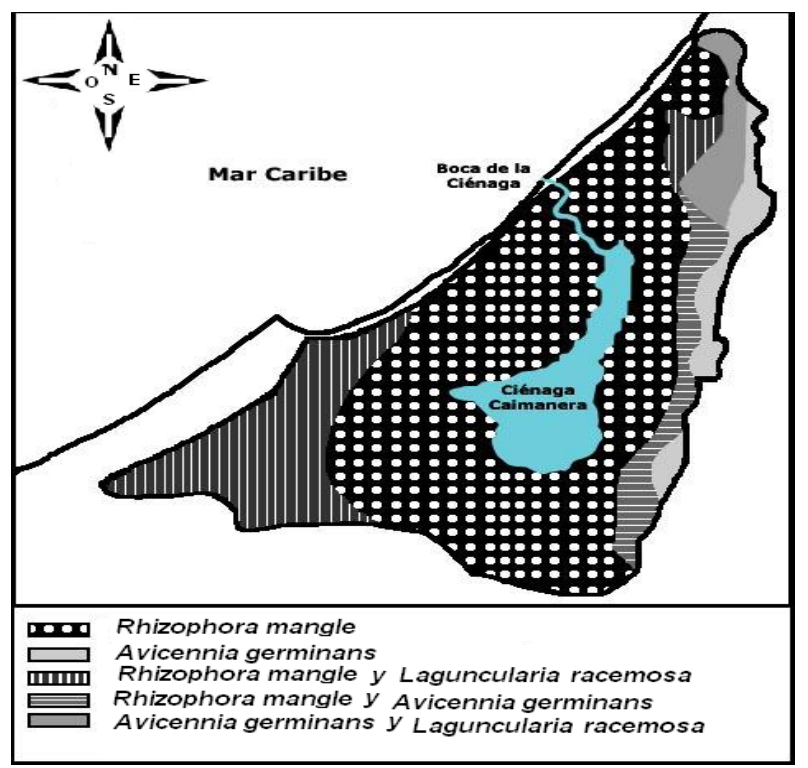

Figura 1. Distribución del arbolado de mangle en la Ciénaga de la Caimanera (Fuente: Modificado de CARSUCRE y Proyecto Manglares del Caribe, 2004)

Según ULLOA-DELGADO et al., (2001) el arbolado de mangle hacia el Oriente y el Nororiente de la Caimanera, está constituido principalmente por latizales, los cuales están representados por arboles maduros de poco porte; sin embargo, en los resultados obtenidos en este estudio a diferencia de lo anterior, se registran zonas en las que existen conjuntos de arboles de mangle negro (Avicennia germinans) y mangle blanco (Laguncularia racemosa) 
maduros, corpulentos y de gran talla, lo que permite inferir que en la Caimanera existen aun pequeñas áreas de manglar que han presentado poca perturbación antrópica, lo cual está relacionado directamente con la poca variabilidad en los parámetros fisicoquímicos de los sustratos en que se desarrollan. No obstante, para el caso del mangle negro el desarrollo de arboles corpulentos podría estar relacionado con el poco valor comercial de los productos extraídos de esta especie, en cuanto a madera se refiere. Además, es importante resaltar que estos árboles presentan abundantes y desarrollados neumatóforos que en última instancia pueden constituir un obstáculo en el proceso normal de regeneración natural ya que limitan la superficie de suelo a la que se adhieren las semillas de estos mangles (INVEMAR, 2005; CASAS-MONROY, 2000; ÁLVAREZ-LEÓN, 2003).

Por otro lado, las visitas de inspección técnica y ocular, permitieron identificar una gran variedad de impactos y/o tensores antrópicos responsable de la pérdida de cobertura vegetal en las áreas de manglar asociadas a las zonas de estudio. Sin embargo, a pesar de que la mayor concentración de áreas boscosas de mangles en el Sur del Golfo está circunscrita alrededor de la Ciénaga la Caimanera, cabe resaltar que los impactos antrópicos responsables de la degradación de cientos de hectáreas de manglar en el departamento, han trascendido significativamente en los manglares de todo el Golfo de Morrosquillo.

En general, según CASAS-MONROY (2000) se considera que la construcción de la carretera Tolú-Coveñas ha influido fuertemente en la degradación progresiva y sostenida en los ecosistemas de manglar, ya que este tipo de infraestructura originó obligatoriamente el asentamiento de poblaciones humanas costeras, las cuales han sido responsable de la tala insostenida de mangles para el acondicionamiento de lotes destinados principalmente a la construcción de viviendas, cabañas, complejos hoteleros y centros recreacionales, en sectores como Boca de la Ciénaga, Punta de Piedra, Palo Blanco, Coveñas, entre otros.

La anterior situación ha ocasionado por mucho tiempo un impedimento en el flujo hídrico normal en los manglares, provocando una disminución en la productividad de estos ecosistemas, debido según SÁNCHEZ-PÁEZ et al., (2004) a que la región del Golfo de Morrosquillo no presenta formaciones delticas importantes que permitan alimentar estas zonas boscosas. Por lo tanto, los drenajes de sus respectivas planicies aluviales están condicionadas por los aportes hídricos de agua continental y las inundaciones que se presentan en temporadas de lluvias, lo que a su vez constituye un problema ya que al interrumpirse el movimiento hídrico desde y hacia el manglar se depositan grandes cantidades de aguas, que en épocas de sequia por efectos de la evaporación ocasionan fenómenos de hipersalinización, que sin lugar a 
dudas inhiben la regeneración natural de los mangles y estimulan en arboles adultos un estrés biológico que ocasiona muertes masivas por enfermedades y exceso de salinidad.

Se corrobora que la disponibilidad de hábitat como playas, manglar, planos lodosos y humedales, que existen en el sector de la Ciénaga la Caimanera, la convierten en un sitio potencialmente importante para aves marinas, playeras y de hábitos dulceacuícolas y estuarinos; así mismo, para especies terrestres de ecosistemas xerófilos, subxerófilos y bosque seco tropical adyacentes al manglar, que por estar sufriendo procesos de fragmentación y destrucción de hábitat, ha ocasionado el desplazamiento de varias especies de aves características de estos ecosistemas hacia los manglares, tal como lo sugiere la información existente para esta zona (DÍAZ, 1997; BARRETO et al.,1999; ULLOA-DELGADO et al., 2001; FRANCO et al., 2005).

En la Tabla 1 se presenta la composición taxonómica de la avifauna observada a lo largo del manglar Ciénaga la Caimanera y su área de influencia (matorrales xerófilos y subxerófilos adyacentes al manglar, playas, salitrales, ciénagas, caños, playones lodosos, humedales y bordes de carretera). Los muestreos realizados en la Ciénaga la Caimanera, permiten registrar un total de 30 especies, pertenecientes a 17 familias.

Es importante resaltar, que durante los recorridos y los muestreos focales no fue posible registrar la totalidad de las especies residentes y visitantes asociadas al manglar de la Caimanera, ya que no se hicieron muestreos durante la noche, por lo que algunas especies de hábitos nocturnos comunes en los manglares del Caribe colombiano como la garza copetiamarilla (Nyctanassa violacea), pasan desapercibidas, según lo reportado por STILES (1998). Como puede apreciarse, la dominancia de unos grupos con respecto a otros es notable debido a las características del hábitat, en particular abundan aves de hábitos acuáticos como las de la familia Ardeidae la cual presenta el mayor número de especies avistadas.

Tabla 1. Composición taxonómica de la avifauna asociada al manglar de la Ciénaga la Caimanera y su área de influencia

\begin{tabular}{|c|l|l|l|l|}
\hline \multirow{2}{*}{ Familia } & \multicolumn{1}{|c|}{ Nombre científico } & Nombre común & \multicolumn{1}{|c|}{ Hábitat } & Hábito \\
\hline \multirow{4}{*}{ ALCENIDAE } & Ceryle torquata & $\begin{array}{l}\text { martín pescador } \\
\text { grande }\end{array}$ & $\mathrm{Ci}, \mathrm{Mg}, \mathrm{FI}$ & Acuática \\
\cline { 2 - 5 } & Chloroceryle americana & $\begin{array}{l}\text { martín pescador } \\
\text { chico }\end{array}$ & $\mathrm{Ci}, \mathrm{Mg}, \mathrm{FI}$ & Acuática \\
\cline { 2 - 5 } & Chloroceryle inda & $\begin{array}{l}\text { martín pescador } \\
\text { selvático }\end{array}$ & $\mathrm{Mg}, \mathrm{Fl}$ & Acuática \\
\hline \multirow{2}{*}{ ANATIDAE } & Anas discors & $\begin{array}{l}\text { pato careto } 0 \\
\text { barraquete }\end{array}$ & $\mathrm{Mg}, \mathrm{Ci}$ & Acuática \\
\cline { 2 - 5 } & Dendrocygna autumnalis & pisingos & $\mathrm{Mg}, \mathrm{Ci}$ & Acuática \\
\hline ANHINGIDAE & Anhinga anhinga & pato aguja & $\mathrm{Mg}, \mathrm{Ci}$ & Acuática \\
\hline
\end{tabular}




\begin{tabular}{|c|c|c|c|c|}
\hline \multirow{6}{*}{ ARDEIDAE } & Ardea alba & garza real & $\mathrm{Mg}, \mathrm{Ci}, \mathrm{FI}$ & Acuática \\
\hline & Nycticorax nycticorax & guaco común & $\mathrm{Mg}, \mathrm{Ci}$ & Acuática \\
\hline & Egretta caerulea & garza azul & $\mathrm{Mg}, \mathrm{Ci}, \mathrm{Ct}$ & Acuática \\
\hline & Egretta thula & $\begin{array}{l}\text { garcita blanca o } \\
\text { patiamarilla }\end{array}$ & $\mathrm{Mg}, \mathrm{Ci}$ & Acuática \\
\hline & Hidranassa tricolor & garza tricolor & $\mathrm{Mg}, \mathrm{Ct}$ & Acuática \\
\hline & Tigrisoma lineatum & vaco colorado & $\mathrm{Mg}, \mathrm{Ci}$ & Acuática \\
\hline CATHARTIDAE & Coragyps atratus & goleros & $\mathrm{Mg}, \mathrm{Vu}, \mathrm{Za}$ & Terrestres \\
\hline \multirow{3}{*}{ COLUMBIDAE } & Columbina talpacoti & tortolita común & $\mathrm{Mg}, \mathrm{Ct}$ & Terrestre \\
\hline & Columba cayennensis & $\begin{array}{l}\text { paloma } \\
\text { guarumera }\end{array}$ & $\mathrm{Mg}$ & Terrestre \\
\hline & Leptotila verreauxi & torcaza & $\mathrm{Mg}$ & Terrestre \\
\hline CUCULIDAE & Crotophaga ani & garrapatero & $\mathrm{Mg}, \mathrm{Za}$ & Terrestre \\
\hline FRINGILLIDAE & Sicalis flaveola & canario basto & $\mathrm{Mg}, \mathrm{Va}, \mathrm{ZA}$ & Terrestre \\
\hline FALCONIDAE & Milvago chimachima & pigua & $\mathrm{Mg}, \mathrm{Ci}$ & Terrestre \\
\hline ICTERIDAE & Quiscalus mexicanus & maría mulata & $\mathrm{Mg}, \mathrm{Pb}$ & Terrestre \\
\hline PELECANIDAE & Pelecanus occidentalis & pelicano pardo & $\mathrm{Mg}, \mathrm{Ct}, \mathrm{Ci}$ & Marina \\
\hline PHALACROCORACIDAE & Phalacrocorax olivaceus & $\begin{array}{l}\text { pato cuervo } 0 \\
\text { buzo }\end{array}$ & $\mathrm{Mg}, \mathrm{Ci}, \mathrm{Ct}, \mathrm{HD}$ & Marina \\
\hline PICIDAE & Chrysoptilus punctigula & pájaro carpintero & $\mathrm{Mg}, \mathrm{Pb}$ & Terrestre \\
\hline \multirow[t]{2}{*}{ PSITTACIDAE } & Aratinga pertinax & cotorra & $\mathrm{Mg}, \mathrm{Ca}$ & Terrestre \\
\hline & Brotogeris jugularis & perico & $\mathrm{Mg}, \mathrm{Ca}, \mathrm{Va}$ & Terrestre \\
\hline THRAUPIDAE & Thraupis episcocus & azulejo común & $\mathrm{Mg}, \mathrm{ZA}, \mathrm{Pb}$ & Terrestre \\
\hline \multirow[t]{2}{*}{ THRESKIORNITHIDAE } & Eudocimus albus & ibis blanco & $\mathrm{Mg}, \mathrm{Ci}$ & Acuática \\
\hline & Ajaia ajaja & espátula rosada & $\mathrm{Mg}, \mathrm{Ci}$ & Acuática \\
\hline \multirow[t]{2}{*}{ TYRANNIDAE } & Pitangus sulphuratus & chicha fría & $\mathrm{Mg}, \mathrm{ZA}, \mathrm{Pb}$ & Terrestre \\
\hline & Fluvicola pica & viudita común & $\mathrm{Mg}, \mathrm{Ci}, \mathrm{FI}$ & Acuática \\
\hline
\end{tabular}

Hábitat: $\mathrm{Mg}=$ manglar; $\mathrm{Ct}=$ costa; $\mathrm{Fl}=$ fluvial; $\mathrm{Sl}=$ salitrales; $\mathrm{Va}=$ vegetación aledaña; $\mathrm{ZA}=$ zonas abiertas: $\mathrm{Pb}=$ poblaciones cercanas; $\mathrm{HD}=$ Humedales de rio o agua dulce.

\section{Referencias}

AGRÁZ HERNÁNDEZ, C.; NORIEGA TREJO, R.; LÓPEZ PORTILLO, J.; FLORESVERDUGO, F.J.; JIMÉNEZ ZACARÍAS, J.J. 2006. Guía de campo. Identificación de los manglares en México. Universidad Autónoma de Campeche. Disponible en: http://etzna.uacam.mx/epomex/paginas/pdf/Guia Manglar.pdf. Fecha de consulta: 10 de mayo de 2009.

ÁLVAREZ-LEÓN, R. 2003. Los manglares de Colombia y la recuperación de sus áreas degradadas: Revisión bibliográfica y nuevas experiencias. Madera y Bosques, primavera. Instituto de Ecología A.C. Xalapa, México.

BARRETO, M.; BARRERA, R.; BENAVIDES, J.; CARDOZO, E.; HERNÁNDEZ, H.; MARÍN, L.; POSADA, B.; SALVATIERRA, C.; SIERRA, P.; VILLA, A. 1999. Diagnostico ambiental del Golfo de Morrosquillo, Una aplicación de sensores remotos y SIG como contribución al manejo integrado de zonas costeras. Curso AGS-6. Editorial IGAC/CIAF. Punta Rada, Tolú, Colombia.

CASAS-MONROY, O. 2000. Estado de los manglares en Colombia. Instituto de Investigaciones Marinas y Costeras, INVEMAR. Disponible en: http://www.invemar.org.co/redcostera1/invemar/docs//EAMC 2000/INVEMAR INF EA MC 2000 06.pdf. Fecha de consulta: 24 de Noviembre del 2009.

CORPORACION AUTONOMA REGIONAL DE SUCRE, CARSUCRE Y PROYECTO MANGLARES DEL CARIBE. 2004. Plan de manejo integrado zona de uso sostenible manglares Ciénaga de la Caimanera. OIMT/CONIF/MINAMBIENTE. Sincelejo, Sucre. 
DÍAZ, G. 1997. Informe nacional sobre el estado de la Biodiversidad en Colombia. Ecosistemas Marinos y Costeros. Programa de Biodiversidad y Ecosistemas marinos. Instituto de Investigaciones Marinas y Costeras, INVEMAR. Santa Marta, Colombia.

DÍAZ ESCOBAR, Y.; MORENO DOMÍNGUEZ, J. 2008. Recuperación y Manejo de los Manglares en la zona Costera de Sucre. Programa. Conservación, uso, restauración de ecosistema de bosques y otros. Proyecto (C8). Trabajo de grado. Universidad de Sucre. Facultad de Educación y Ciencias. Programa de Biología. Sincelejo, Sucre.

DUNN, E.H.; BART, J.; COLLINS, B.T.; CRAIG, B.; DALE, B.; DOWNES, C.M.; M. FRANCIS, C.M.; WOODLEY, S.; ZORN. P. 2006. Monitoring bird populations in small geographic areas. Special Publication. Canadian Wildlife Service. Ottawa, Ontario.

FRANCO-MAYA, A. M.; BRAVO, G. A. 2005. Áreas importantes para la conservación de las aves en Colombia. En: BOYLA, K. y ESTRADA, A. (Eds.) Áreas importantes para la conservación de las aves en los Andes tropicales; sitios prioritarios para la conservación de la biodiversidad. Birdlife Internacional y Conservación Internacional. Quito, Ecuador.

GIL-TORRES, W. 1998. Dinámica de crecimiento de los manglares del Caribe continental de Colombia: Proyecto PD 171/91 Rev. 2 Fase II (Etapa I) Conservación y manejo para el uso múltiple y el desarrollo de los manglares de Colombia, MAVDT/ACOFORE/OIMT. Bogotá, Colombia.

INGEOMINAS. 1998. Geomorfología y aspectos erosivos del litoral Caribe colombiano. Geomorfología y aspectos erosivos del litoral Pacífico colombiano. Publicación geológica especial. Bogotá, Colombia.

INSTITUTO DE INVESTIGACIONES MARINAS Y COSTERAS, INVEMAR. 2005. LoS ecosistemas del Mar Caribe colombiano y de sus costas. Encuentros ambientales. Universidad del Norte. Barranquilla, Colombia.

MINISTERIO DEL MEDIO AMBIENTE. 2002. Uso sostenible, manejo y conservación de los ecosistemas de manglar en Colombia. Programa nacional. Dirección General de Ecosistemas. Bogotá, Colombia.

NARANJO, L.G. 1979. Las aves marinas del Caribe colombiano: Taxonomía, zoogeografía y anotaciones ecológicas. Trabajo de grado. Fundación Universidad de Bogotá Jorge Tadeo Lozano. Facultad de Biología Marina. Bogotá, Colombia.

SÁNCHEZ-PÁEZ, H. y R. ÁLVAREZ-LEÓN. 1997. Diagnóstico y zonificación preliminar de los manglares del Caribe de Colombia. OIMT/ MAVDT. Bogotá, Colombia.

SÁNCHEZ-PAÉZ, H.; ULLOA-DELGADO, G.; ÁLVAREZ-LEÓN, R.; GIL-TORRES, W.; SÁNCHEZ-ALFÉREZ, A.; GUEVARA-MANCERA, O.; PATIÑO-CALLEJAS, L.; PÁEZPARRA, F. 2000. Hacia la recuperación de los manglares del Caribe de Colombia. MAVDT/ACOFORE/OIMT. Bogotá, Colombia.

SÁNCHEZ-PÁEZ, H.; ULLOA-DELGADO, G.; TAVERA-ESCOBAR, H. 2004. Manejo integral de los manglares por comunidades locales, Caribe Colombiano. Proyecto PD 60/01 Rev. 1(F) Manejo sostenible y restauración de los manglares por comunidades locales del Caribe de Colombia. Bogotá, Colombia. 
STILES, F.G. 1998. Aves endémicas de Colombia en: Chaves, M.E y Arango, N. Informe Nacional sobre el Estado de la Biodiversidad en Colombia (Vol. I). Instituto de Investigación en Recursos Biológicos Alexander Von Humboldt/PNUMA/Ministerio de Medio Ambiente. Bogotá, Colombia.

ULLOA-DELGADO, G.; GIL-TORRES, W. 2001. Caracterización, diagnostico y zonificación de los manglares en el departamento de Sucre. Corporación Autónoma Regional de Sucre, CARSUCRE. Sincelejo, Sucre, Colombia. 